\title{
Interaction of Piriformospora indica (Serendipita indica) with ZnO Nanoparticles and their Impact on Brassica oleracea
}

\author{
Ajit Varma ${ }^{1 *}$, Manpreet Kaur Attri ${ }^{1}$, Hemesh Joshi ${ }^{1}$, Surbhi Dabral' ${ }^{1}$, Shubhangi Mahajan ${ }^{1}$, Neelam Patel $^{2}$ and \\ Manika Khanuja ${ }^{3}$ \\ ${ }^{1}$ Amity Institute of Microbial Technology, Amity University, Noida, Uttar Pradesh, India \\ ${ }^{2}$ Centre for Protected Cultivation, IARI, New Delhi, India \\ ${ }^{3}$ Centre for Nanoscience and Nanotechnology, Jamia Milia Islamia, New Delhi, India \\ *Corresponding Author: Ajit Varma, Professor, Amity Institute of Microbial Technology, Amity University, Noida, Uttar Pradesh, India.
}

Received: June 03, 2019; Published: June 18, 2019

DOI: $10.31080 /$ ASPS.2019.03.0316

Piriformospora indica (Serendipita indica) promotes plant growth and also value addition to the host. It obviates the biotic and abiotic stresses. Interaction with $\mathrm{ZnO}$ nanomaterials led to enlarged colony size, hyphal and spores diameter. Nano embedded fungal biomass influenced early flowering and bigger heads.

Piriformospora indica, Verma, Varma, Kost, Rexer and Franken is a potential candidate to serve as bio fertilizer, bio protector, bio regulator, bio herbicide/weedicide and an excellent source for the hardening of the tissue culture raised crops/plants. $P$. indica tremendously improves the growth and overall biomass production of diverse hosts, including legumes, medicinal and economically important hosts. The fungus also has the potential to act as bio protectant against fungus root pathogens and soil insects. Pronounced growth promotional effect was also seen with terrestrial orchids. The fungus promises to be a potential orchid mycorrhizal fungus [1]. This symbiont interacts with one thousand and one land plants.

There is a new name i.e. "Group B" given to Sebacinales which belongs to Sebacinaceae. The molecular phylogenetic analysis also proves that Piriformospora is taxonomically similar with Serendipita. So it is proposed that Piriformospora can be merged with Serendipita. This is a telemorph i.e. sexual stage where as Piriformospora is anamorph i.e. asexual stage. The name for this new genetic concept should be Serendipita [2]. Sebacinales's phylogenetic position with in Basidiomycota.

Mycobiont was cultivated in batch culture containing 4\% jaggery (sugarcane) solution as sole carbon and energy source (Patent number: 944/DEL/2012 dt:27-03-12) [3]. Nano ZnO was synthesized.

$\mathrm{ZnO}$ nanorods were prepared by a mechanical-assisted thermal decomposition process. Zinc acetate dihydrate $\left[\mathrm{Zn}\left(\mathrm{CH}_{3} \mathrm{COO}\right) 22 \mathrm{H}_{2} \mathrm{O}\right]$ was used as precursor for synthesis of zinc oxide nanorods. In the synthesis process, zinc acetate dihydrate ( $5 \mathrm{~g}$ ) was ground in a mortar and pestle for $45 \mathrm{~min}$ then loaded into an alumina crucible, which was then heated in a programmable muffle furnace (at ramp rate of $40 \mathrm{C} / \mathrm{min}$ ) at $2750 \mathrm{C}$ ) for $4 \mathrm{~h}$ to obtain $\mathrm{ZnO}$ nanopowders. The obtained powders were then washed twice with distilled water, followed by drying in an memmert type oven at $800 \mathrm{C}$ for 8 hours [4]. The nanoparticles were spherical in shape and 9.6-25.5 $\mathrm{nm}$ in size. The fungus was incubated with $20 \mu \mathrm{g} / \mathrm{ml}$ with nanomaterial for 7 days and constant shaking conditions (100rpm) at $30^{\circ} \mathrm{C}$. This fungus biomass was filtered and mixed with Brassica seeds at a rate of $2 \%(\mathrm{~W} / \mathrm{v})$. Seeds were grown in field under protected environmental conditions at Indian Agricultural Research Institute, New Delhi.

The mycobiont on incubation at 28 Cmade rhythmic rings on agar fortified medium (Figure 1a) and Round globular dense colonies in broth (Figure 1b).

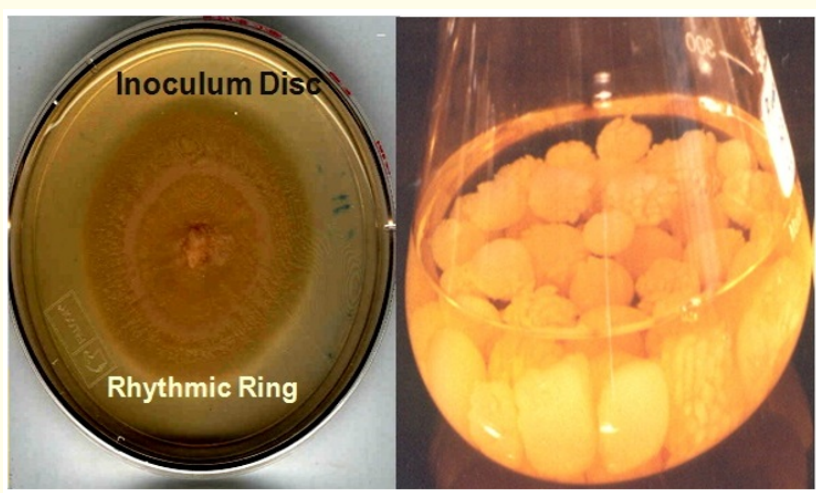

Figure 1: Mass multiplication on cheap, reproducibly and reliable Jaggery (Gur) as sole Carbon energy source.

The fungus makes pear shaped bunches of spores (Figure 2a). Hyphae highly interwoven, often adhere together and gave the 
appearance of simple cords. New branches emerged irregularly, and at regular intervals the hyphal wall showed some external deposits that stained with toluidine blue (Figure $2 b$ ).

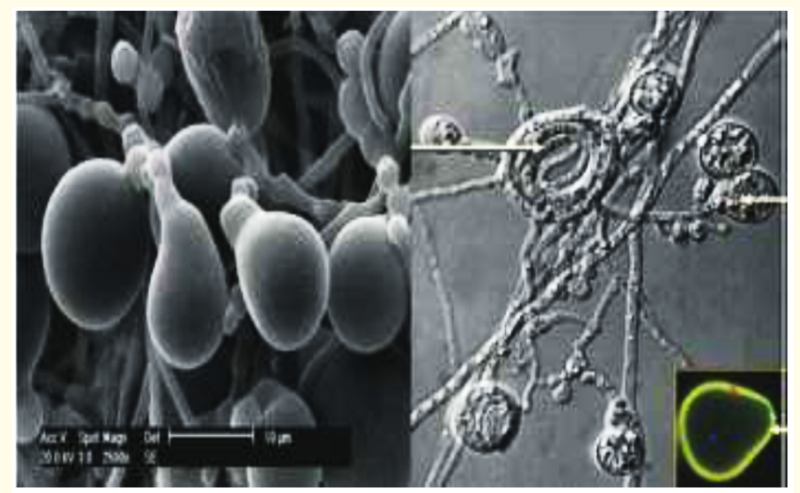

Figure 2: Fungus Showing Fluorescent Pear Shaped Spores.

Figure 3a was made using Confocal microscope (Model No. NIKON- A1) and were stained with W5 Wheat germ dye (Figure $3 b)$. Interestingly the spores in nano treated medium were enlarged spores, thick walled, thick hyphae and less vacuole as compared to non treated fungal biomass (Figure 4). Although the plant biomass was healthier in $\mathrm{Pi}$ and $\mathrm{ZnO}-\mathrm{Pi}$ treated plants as compared to control. However, early flowering and fruiting were recorded when the seeds were treated with Nano ZnO (Figure 5). Consequently the nano treated plants produced large flower heads (Figure 5 and $6)$. Average weight of each nano treated flower head was around 787, Piri alone was 718 and control 609 grams, respectively.
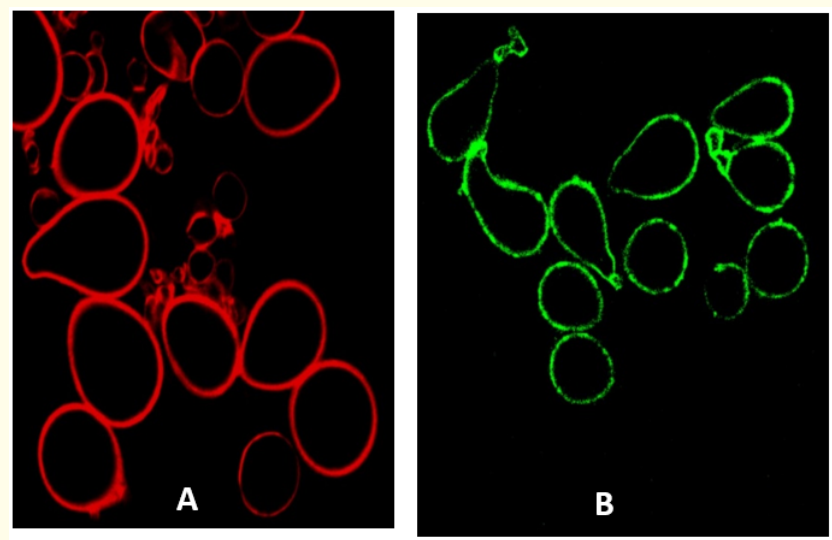

Figure 3: Confocal Microscopy.

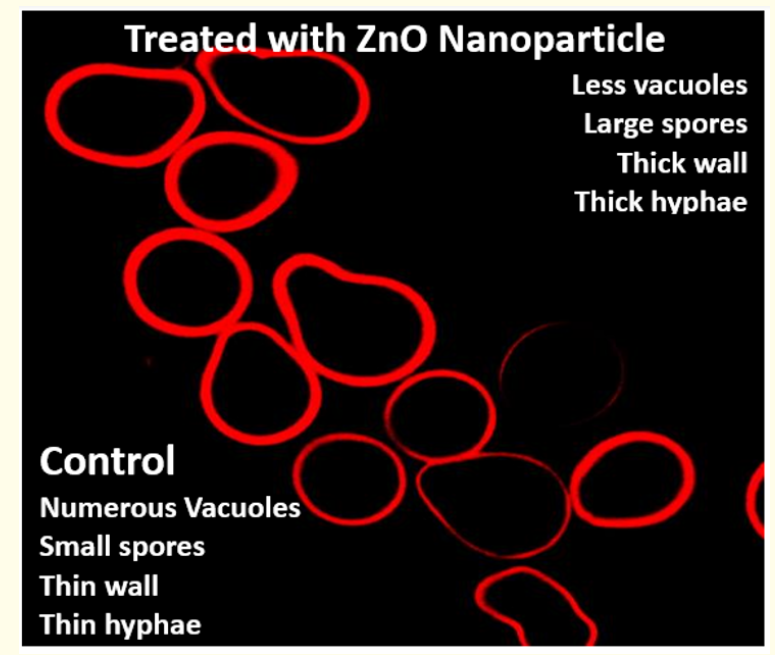

Figure 4: Characteristics of the treated and untreated spores.

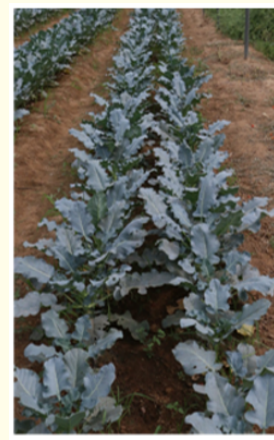

Control

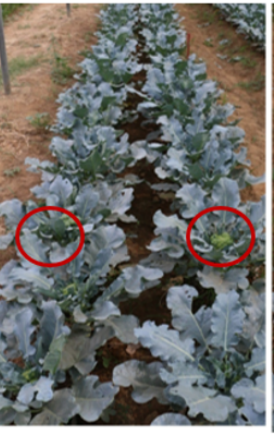

$P$. indica $+\mathrm{ZnO}$

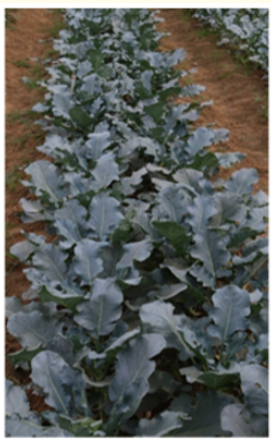

P. indica
Figure 5: Protected Field Cultivation at IARI, New Delhi.

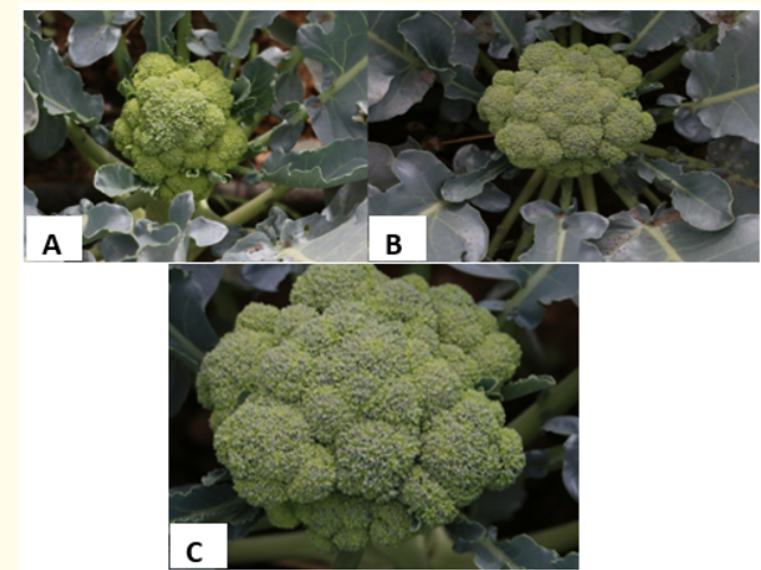

Figure 6: A view of Flower Heads. Photographed after 6 weeks. A: Control, B. P. indica, C. Nano treated. 
We conclude that Broccoli seeds treated with nano Zinc material promotes the plant growth, early flowering and early setting of flower heads. The secondary metabolites i.e. polyphenol, Glucosinolate, 4-hydroxy and methoxy glucobrassicin, glucoerucin, gluconasturtin is increased by $\mathrm{b} 10-12 \%$ in treated broccoli as compared to control. At present we do not know the mechanism involved for enlarge spore size and there influence on flower head.

Authors are thankful to NASF (ICAR), DST-FIST and Nano Mission- DST for partial financial assistances.

\section{Bibliography}

1. Savita V., et al. "Piriformospora indica, gen. et sp.nov., a new root-colonizing fungus”. Mycologia USA 90 (1998): 896-903.

2. Michael W., et al. "Sebacinales-one thousand and one interactions with land plants". New Phytologist 211 (2016): 20-40.

3. Soubhagya B., et al. "Interaction of Piriformospora indica with Azotobacter chroococcum". Nature Scientific Reports 5 (2015): 13911-13924.

4. Tamanna B., et al. "Biosynthesise of zinc oxide nanoparticles from Azadirachta indica for antibacterial and photocatalytic applications". Materials Science in Semiconductor Processing 32 (2015): 55-61.

Volume 3 Issue 7 July 2019

(C) All rights are reserved by Ajit Varma., et al. 